\title{
Ciudad y ciudadanía. Un análisis de los planes estratégicos de desarrollo urbano desde la perspectiva de género
}

\author{
Carmen INNERARITY GRAU \\ Universidad Pública de Navarra \\ Ana SANCHO MARTINEZ \\ Asociación Bilbao Metropoli-30
}

Recibido: 23.07 .2014

Aceptado: 30.01.2015

\begin{abstract}
RESUMEN:
Este estudio se enmarca dentro del análisis de las políticas de planificación urbana desde la perspectiva de género. Parte de la idea de que la inclusión de las mujeres en el espacio público podría estar chocando con un diseño de las ciudades en el que aún pesa mucho la vieja división sexual del trabajo, según el cual a los hombres corresponde una zona de la ciudad (en la que ellos trabajan) y a las mujeres, otra (en la cual ellas residen), haciendo muy difícil, no sólo la inclusión de las mujeres en pie de igualdad, sino también la conciliación tanto por parte de varones como de mujeres.

A partir de aquí, el artículo examina en qué medida los planes estratégicos y urbanísticos de ciudades españolas (Bilbao, Barcelona, Zaragoza, Málaga y Jaén) han incluido la perspectiva de género. Concretamente, analiza si los planes recogen las recomendaciones dictadas desde los años noventa por los organismos internacionales -fundamentalmente el Programa de las Naciones Unidas para los Asentamientos Humanos, ONU-Habitat- de cara a la inclusión del género de forma transversal en las políticas de planificación urbana con el fin de hacer "ciudades conciliadoras".
\end{abstract}

Palabras clave: Urbanismo; Planificación urbana; Género; Políticas públicas; Ciudadanía

\section{City and citizenship. An analysis of the strategic plans of urban development from the perspective of gender}

\begin{abstract}
This study focuses on urban planning policies from the standpoint of gender. It stems from the idea that the inclusion of women in public space could be clashing with a design of cities in which the old sexual division of work still weighs heavily. According to it an area of the city corresponds to men (where they work), and another area of the city corresponds to women (where they live). Not only is this an obstacle for the inclusion of women on equal conditions, but also for the conciliation of family and work life.
\end{abstract}


Based on this assumption, the article examines to what degree the strategic and urban plans of Spanish cities (Bilbao, Barcelona, Zaragoza, Malaga and Jaen) take into consideration the gender perspective. Specifically, the study analyses if these plans include the recommendations made by international organisations since the 1990s - mainly the United Nations Human Settlements Programme (UNHabitat) - as regards the transversal inclusion of gender in public policies of urban planning, with the aim of making "conciliatory cities."

Key words: Urbanism Urban planning; Gender; Public policies; Citizenship

Nuestro análisis parte de la crítica feminista a la radical separación entre lo público y lo privado y la atribución de uno y otro espacio a varones y mujeres, respectivamente (Pateman, 1988; Young, 1990; Kymlicka, 1996). Y es que, a pesar de los cambios en los papeles atribuidos a cada uno de los géneros, el acceso de las mujeres al espacio público - a la ciudad, como su materialización por excelenciapodría estar chocando con un diseño desagregado de las ciudades en el que aún pesa mucho la vieja división sexual del trabajo según el cual a los hombres corresponde una zona de la ciudad (en la que ellos trabajan) y a las mujeres, otra (en la cual ellas residen y se ocupan de las tareas relativas al cuidado). Son ciudades dispersas, en las que la separación de espacios y funciones sociales hace difícil -aunque sólo sea por el tiempo que supone- no solo la inclusión de las mujeres en condiciones de igualdad, sino también que tanto varones como mujeres transiten de uno a otro espacio, de una a otra función, es decir, la conciliación. De ahí la necesidad de incorporar la perspectiva de género en las políticas de planificación urbana en todos los niveles de gobernanza.

Este trabajo examina, en primer lugar, las principales recomendaciones para la planificación de las ciudades con perspectiva de género elaboradas a partir de la década de los noventa por Organismos Internacionales, fundamentalmente, el Programa de las Naciones Unidas para los Asentamientos Humanos, ONU-Habitat y, en el ámbito europeo, la Carta Europea de las Mujeres en las ciudades. De ellos se pueden extraer una serie de indicaciones de cara a la inclusión del género de forma transversal en las políticas de planificación urbana, que, en último término, serán los ayuntamientos quienes deban poner en práctica. Por eso, pretendemos analizar en qué medida los planes locales se hacen eco de las directrices de los organismos internacionales estudiados, apostando, así, por el diseño de ciudades conciliadoras. Frente a esas ciudades dispersas que separan espacios y funciones, es preciso -y este será una de las tesis de este trabajo- repensar la ciudad desde la heterogeneidad, la mezcla, con el fin de diseñar ciudades que integren, no que separen, la diferencia. Existe todo un abanico de diferencias de clase, de edad, culturales, que afectan también a las mujeres, estableciendo desigualdades entre ellas y que deben ser tenidas en cuenta en la elaboración de las políticas de planificación urbana, para atender, por ejemplo, a las necesidades específicas de la 
juventud o de la tercera edad, o a la situación particular de las mujeres en los barrios con una elevada concentración de población inmigrante (Innerarity, 2007; 2014), o para evitar la formación de guetos, con toda la problemática que ello implica (Innerarity, 2009). Sin embargo, la necesidad de delimitar nuestro objeto de estudio, nos lleva a centrar nuestro análisis en las diferencias de género sin atender a otras categorías de diversidad.

\section{LA CIUDAD: CIUDADANÍA UNIVERSAL/CIUDADANÍA DE VARONES/CIUDADANÍA DIFERENCIADA}

El marco teórico de nuestro trabajo es la crítica realizada por el movimiento feminista a la separación entre lo público y lo privado que está en el núcleo de la sociedad liberal. Como señala Carol Pateman, "en realidad, esa dicotomía es aquello sobre lo que trata, fundamentalmente, el movimiento feminista" (Pateman, 1996: 2). El profundo compromiso liberal con el igualitarismo lleva a considerar al individuo abstracto, al margen de sus peculiaridades de género, etnia o religión, como sujeto de la ciudadanía. La ciudadanía universal, la igualdad, supone, así, la universalidad de la ciudadanía en cuanto trascendencia de la particularidad y la diferencia, que deben quedar en el ámbito privado. La división entre espacio público y espacio privado es, así, un elemento fundacional del proyecto liberal y su aspiración a la igualdad, que pasa por establecer un mundo de barreras, un mundo de segregación, fundamentalmente entre lo público -igualitario por universal- y lo privado -diferente por particular. La esfera pública es la esfera del interés general, de la racionalidad, mientras que la esfera privada lo es del interés particular, la emoción y el cuidado, atribuidos uno y otro a varones y mujeres, respectivamente.

Pero la ceguera respecto a lo privado y lo particular impide considerar de manera suficiente el hecho de que muchas desigualdades en la participación en el espacio público hunden sus raíces en circunstancias específicas que afectan a las vidas de las personas en el ámbito privado. Concretamente, la igualdad formal de las mujeres en la esfera pública, en sus manifestaciones políticas, sociales y económicas queda disminuida por la carga de sus responsabilidades en la vida privada. El espacio público, teóricamente ciego a las diferencias, oculta la realidad del "patriarcalismo liberal" (Pateman, 1996: 35), de una sociedad estructurada a partir de relaciones jerárquicas que tienen su raíz en la esfera doméstica privada.

Y aquí radica un segundo aspecto -junto a esa separación de espacios y la atribución de lo público a los varones y lo privado a las mujeres- de la crítica feminista a la sociedad liberal que consideramos relevante para nuestro estudio y que es la denuncia de la falsa neutralidad del espacio público y de la ciudadanía. La 
igualdad que pretende lograr la sociedad liberal mediante la neutralidad de sus instituciones puede significar dos cosas: tratar de modo igual o tratar como iguales. El problema es que "cuando varones y mujeres son tratados de modo igual, el varón resulta ser la norma frente a la cual la mujer es peculiar, incompleta y diferente" (Phillips, 1993: 45). De esta forma, "los criterios aparentemente universales que rigen la sociedad civil son, en realidad, los criterios que se asocian con la noción liberal del individuo varón, que se presenta como la noción del individuo" (Pateman, 1996: 35). El ideal de ciudadano se configura a partir de la consideración de "la persona individual y totalmente móvil, sin tomar en consideración los vínculos y las condiciones sociales de su existencia y su identidad" (Beck y BeckGernsheim, 2001: 22), un individuo que puede salir al espacio público porque cuenta con una mujer que lleva a cabo las tareas relacionadas con el cuidado. Las experiencias y las necesidades asociadas a esas responsabilidades del ámbito privado son vistas como lo diferente, lo particular aquello cuya consideración impediría una sociedad de iguales.

De ahí el mito de la supuesta neutralidad de ese espacio público y de las instituciones y normas que lo configuran, denunciado por la política de la diferencia. En palabras de Iris M. Young, "fundado por hombres, el Estado moderno y el dominio público de la ciudadanía presentó como valores y normas universales aquellas que habían derivado de la experiencia específicamente masculina" (Young, 1996: 102). La perspectiva imparcial es, de hecho, un mito que esconde el sesgo de género de las instituciones y los patrones de reconocimiento, que responden a la identidad y las necesidades del varón. De modo semejante a lo que sucede con otras diferencias identitarias, la pretendida neutralidad oculta "la sujeción de las mujeres a los hombres dentro de un orden aparentemente universal, igualitario e individualista" (Pateman, 1996: 32). Por eso, cualquier intento de "entrar" en el espacio público choca con las barreras simbólicas de un ámbito pensado por y para los varones, de un ámbito que no les pertenece como algo propio sino en el que entran como intrusas. Significa que hombres y mujeres se incorporan a la ciudadanía y entran en la ciudad de formas diferentes: los varones lo hacen en calidad de soldados y trabajadores, las mujeres, como madres y cuidadoras.

La división de esferas y la falsa neutralidad de la ciudadanía encuentran su reflejo en la planificación de las ciudades. A pesar de los cambios en las relaciones familiares, el diseño de las ciudades sigue siendo, en gran medida, heredero de una división sexual del trabajo que considera que actividad doméstica y actividad productiva son independientes y deben estar situadas, por tanto, en espacios y horarios distintos. Así, la ciudad ubica en lugares diferentes la realización de las dos funciones principales: residir y trabajar, de forma que resulta muy difícil o, al 
menos, exige mucho tiempo trasladarse de uno a otro para ocuparse de ambas actividades, es decir, conciliar.

Por eso, una reinterpretación de la ciudadanía y la ciudad desde el punto de vista de género exige una deconstrucción de la dicotomía de espacios, siguiendo la crítica feminista al pensamiento binario, que genera una lógica de subordinación, de jerarquía, de desigualdad, de segregación. No se trata solamente de entrar en la ciudad, de acabar con la tradicional atribución segregada de espacios, sino que es preciso dar un paso más haciendo la ciudad más hospitalaria con la diferencia, concretamente, con la diferencia de género. Para ello es necesario diseñar una ciudad que atienda a las necesidades que se derivan de las experiencias específicas de las mujeres, una ciudad que facilite el tránsito de varones y mujeres de uno a otro espacio, una ciudad compacta que permita trabajar y cuidar como actividades que no se ubican en compartimentos ni en individuos estancos; una ciudad conciliadora y corresponsable, que permita la coordinación de las tareas laborales y domésticas considerando las cadenas de actividad como la clave principal, para llegar poco a poco a una sostenibilidad urbanística (Sánchez de Madariaga, 2009: 591).

Pensar la ciudad desde la diferencia implica diseñar espacios públicos heterogéneos en los que se reconozcan las distintas categorías de diversidad como irreductibles, "que renuncien tanto a la ambición moderna de borrar del mapa las diferencias, como a la tendencia postmoderna que lleva a la fosilización de dichas diferencias mediante la separación y el distanciamiento de unos y otros. Hablamos de lugares públicos que reconozcan el valor original y enriquecedor de la diversidad, y animen a las personas diferentes a entablar un diálogo que valga la pena" (Bauman, 2006: 56). Pensar el espacio urbano de forma inclusiva de la diversidad exige atender "en igualdad de condiciones todas las demandas, sin decisiones a priori, que excluyan la experiencia de las personas como fuente fundamental de conocimiento en las decisiones urbanas" (Muxi y otros, 2011: 107). La experiencia que los varones tienen del espacio es diferente de la de las mujeres, por eso es necesario tener atender a ambas en la planificación (Muxí y otros, 2011: 122; 2014: 27). Es preciso, en definitiva, construir ciudades complejas y mixtas, que sean el reflejo de una sociedad que es diversa y que, aceptando y partiendo de esa diversidad, puedan diseñar sus espacios de manera inclusiva respondiendo a las circunstancias específicas tanto de varones como de mujeres.

\section{PRINCIPALES RECOMENDACIONES SOBRE URBANISMO CON PERSPECTIVA DE GÉNERO}

La necesidad de reorganizar el espacio público de forma que rompa esa dicotomía de espacios y funciones y que sea realmente inclusivo para las mujeres entra en la 
agenda política, principalmente, a partir de mediados de la década de los noventa. Desde entonces diversas instituciones y organismos internacionales comienzan a elaborar una serie de declaraciones y a sugerir medidas que proponen la inclusión del género de forma transversal en las políticas públicas de planificación de las ciudades. En este apartado expondremos las principales recomendaciones del Programa Habitat de la Organización Nacional de las Naciones Unidas y la Carta Europea de las Mujeres en la ciudad, promovida por la Comisión de la Unión Europea. La importancia de estos dos programas radica en el hecho de ser la primera vez que incluyen la perspectiva de género en el urbanismo, cada una en su ámbito, abriendo una línea de trabajo que llega hasta nuestros días. Pretendemos sintetizar cuáles son las principales recomendaciones propuestas para el diseño de ciudades integradoras, con el fin de analizar posteriormente en qué medida los planes urbanos recogen esas indicaciones.

\subsection{Conferencias de Naciones Unidas sobre Asentamientos Humanos. Habitat I y II}

La I Conferencia de Naciones Unidas sobre Asentamientos Humanos tuvo lugar en Vancouver (Canadá) en 1976. Esta Conferencia dio lugar a la elaboración del Informe Mundial sobre los Asentamientos Humanos y a la aparición del Centro de las Naciones Unidas para los Asentamientos Humanos (CNUAH), precursor del actual ONU-HABITAT (Programa de las Naciones Unidas para los Asentamientos Humanos).

La Segunda Conferencia de las Naciones Unidas sobre los Asentamientos Humanos (Habitat II) se celebró en Estambul en 1996. Fruto de la conferencia Habitat II fue la aprobación de dos documentos: la Declaración de Estambul sobre los Asentamientos Humanos, que contiene los objetivos y compromisos adoptados por los estados miembro de la ONU para mejorar las condiciones de los asentamientos humanos, y el Programa Habitat, que recoge el Plan de Acción Mundial: Estrategias para la Aplicación, también conocido como Agenda Habitat. Desde entonces, el Programa Habitat ha constituido una referencia fundamental a la hora de establecer prioridades para el desarrollo urbano sostenible adecuado a las necesidades de las personas, una de ellas, la de integrar la perspectiva de género.

Entre los objetivos del Plan de Acción Mundial destaca el acceso universal a una vivienda adecuada. Desde la adopción de la Declaración Universal sobre los Derechos Humanos en 1948, el acceso a una vivienda ha sido reconocido como un componente importante del derecho a un nivel de vida digno por el que los estados deben velar. Pero los gobiernos son conscientes de que no basta con un compromiso general para favorecer el acceso a la vivienda, sino que declaran expresamente la necesidad de atender a la situación específica de las mujeres, ya que a menudo 
disponen de menos recursos como propiedad, crédito, capacitación y tecnología. De alguna manera, los gobiernos manifiestan un compromiso con la política de la diferencia que apunta a la necesidad de llevar a cabo políticas "encarnadas" que, más allá del objetivo general de garantizar el derecho universal a la vivienda, atiendan a las situaciones específicas en las que se encuentran las mujeres. Para ello, frente a la universalidad, la abstracción, la racionalidad que, de acuerdo con los postulados liberales, deben regir el espacio público y el diseño de las instituciones que lo ordenan, es preciso escuchar las voces de las mujeres, impulsando medidas que faciliten su participación.

Esta apuesta por una política de la diferencia se manifiesta de manera especial en dos principios de Habitat: el relativo al papel de la mujer ${ }^{1}$ y el referente a la planificación ${ }^{2}$. En ellos se recogen dos consideraciones fundamentales que debe tener en cuenta el diseño de los asentamientos humanos en relación con el género. En primer lugar, la situación de desigualdad que afecta a la mujer y la necesidad de remover los obstáculos que impiden su plena participación en el espacio público y, en segundo lugar, la exigencia de considerar las necesidades de la familia y favorecer su preservación con vivienda adecuada y acceso a los servicios básicos.

En el proceso de implementación de las conclusiones de Habitat II "con el fin de promover la igualdad de género en todos sus esfuerzos y programas" (ONUHABITAT, 2010), cabe destacar una serie de hitos importantes que reseñamos a continuación.

En primer lugar, uno de los primeros pasos en la aplicación de las recomendaciones de Habitat fue la creación en 1997 de la Comisión de Naciones Unidas para los Asentamientos Humanos con el fin de dar seguimiento al progreso

\footnotetext{
1 "La mujer tiene un papel de primer orden que desempeñar en el logro de asentamientos humanos sostenibles. No obstante, a causa de diversos factores, entre los que figura la persistente y creciente carga de la pobreza para las mujeres y la discriminación en razón del género, la mujer tropieza con obstáculos particulares cuando trata de obtener una vivienda adecuada y de participar plenamente en la adopción de decisiones relativas a los asentamientos humanos sostenibles. La emancipación de la mujer y su participación plena y en condiciones de igualdad en la vida política, social y económica, la mejora de la salud y la erradicación de la pobreza son indispensables para lograr la sostenibilidad de los asentamientos humanos" (Habitat, 1996).

2 "Al planificar los asentamientos humanos debe tener presente el papel constructivo de la familia en el diseño, el desarrollo y la ordenación de esos asentamientos. La sociedad deberá facilitar, cuando proceda, todas las condiciones necesarias para su integración, reunificación, conservación, mejora y protección en viviendas adecuadas que tengan acceso a los servicios básicos y a medios de vida sostenibles" (Habitat, 1996).
} 
global en la implementación de la Agenda Habitat y a las condiciones y tendencias urbanas globales. Fue el llamado Observatorio Urbano Global, una amplia base mundial de datos de ciudades en el mundo. Además, cinco años después de Estambul, se llevó a cabo un proceso de revisión oficial que comenzó en octubre de 1999 con el lanzamiento de la Guía para los informes de país, con el título Indicadores Urbanos Estambul + 5 (2001), desarrollada por ONU-Habitat. Esta Guía fue elaborada a partir de los informes realizados por los países sobre el cumplimiento de los objetivos de la Agenda Habitat, incluyendo un balance de género, tras un proceso participativo en el que las redes de mujeres se movilizaron ampliamente. La Declaración sobre ciudades y otros asentamientos humanos en el nuevo milenio, recoge las conclusiones de la sesión de Estambul +5 , reafirmando todos los compromisos de género adquiridos previamente.

En esta misma línea, la promoción de la igualdad de género y el empoderamiento de las mujeres es el tercero de los ocho Objetivos de Desarrollo del Milenio consensuados por la comunidad mundial en el año 2000, desarrollado en el documento Igualdad de género para ciudades más inteligentes. Desafios y avances (2010), que muestra cómo, a pesar de que ya han pasado años desde Habitat, la ONU no ha olvidado el compromiso adquirido con la perspectiva de género, sino que continúa "identificando y monitoreando desigualdades, incluyendo aquellas de naturaleza relacionada con el género" (ONU-HABITAT, 2010) y promoviendo buenas prácticas, convencida de que "las ciudades se convierten en lugares privilegiados para la promoción de esta igualdad" (ONU-HABITAT, 2010). Consciente de ello, ONU-HABITAT ha elaborado un Plan de Acción de Igualdad de Género para los años 2008 a 2013 con el fin de coordinar las perspectivas dominantes sobre género en todos los programas, políticas y actividades. El plan señala seis áreas de acción, cada una de las cuales coincide con las seis áreas focales del Plan Institucional Estratégico a Medio Plazo (2008-2013) de la agencia: defensa y monitorización de la igualdad de género en las ciudades, participación de las mujeres en el gobierno local, programas de gestión del suelo y administración de vivienda sensibles al género, acceso a servicios urbanos acorde con las necesidades específicas de las mujeres, facilitar a las mujeres el acceso a la financiación para vivienda, capacitación del personal para el desarrollo de herramientas y aplicación de directrices sobre género (ONU-HABITAT, 2010).

Desde entonces y hasta la actualidad, ONU Habitat publica toda una serie de documentos sobre estos temas que reflejan cómo el compromiso con la integración de la perspectiva de género en la planificación urbana sigue vivo ${ }^{3}$. De especial

3 En 2014 (Gender Issue Guide: Gender Responsive Urban Research and Capacity Development, Women in Post-Conflict Settlement Planning, Gender Equality Action Plan: 
interés es la publicación Planificación y diseño de una movilidad urbana sostenible: Informe Mundial sobre Asentamientos Humanos 2013. El informe busca poner de relieve los retos del transporte a que se enfrentan las ciudades en todo el mundo e identificar ejemplos de buenas prácticas para abordar estos desafíos de una manera más sostenible. El Resumen Ejecutivo del Informe señala que "en todas las sociedades del mundo, los hombres y las mujeres a menudo suelen desempeñar diferentes roles. Hay razones sobradas para incorporar las cuestiones de género en la filosofía de trabajo de las organizaciones del transporte urbano" (ONUHABITAT 2013). Una vez más, Naciones Unidas subraya la necesidad de atender al hecho de que las mujeres tienen necesidades específicas derivadas del peso que aún tiene en nuestras sociedades la tradicional división de funciones, que se plasma también en el diseño de las ciudades. De ahí que sea necesario no sólo atender al punto de vista de las mujeres en la planificación urbana, sino también, tomar medidas -en este caso relativas al transporte- que faciliten el tránsito de un espacio a otro.

En conclusión, la Declaración de Estambul del año 1996 constituyó una llamada de atención sobre la necesidad de lograr el compromiso de los gobiernos para integrar la perspectiva de género en las políticas relativas a los asentamientos humanos. Desde entonces, como hemos visto, el compromiso de Naciones Unidas con una política de planificación urbana que atienda a la diferencia de género ha sido constante.

\subsection{Carta Europea de las Mujeres en la Ciudad}

La principal referencia sobre el urbanismo con perspectiva de género en el ámbito de la Unión Europea es la Carta Europea de las Mujeres en la Ciudad, que "pretende concebir una nueva filosofía en el planeamiento urbano que tenga en cuenta las necesidades y las diferentes expectativas de los ciudadanos, tanto de las mujeres como de los hombres" (Carta, 1996). Es una propuesta de investigaciónacción planteada en el marco del Programa para la Igualdad de Oportunidades de las Mujeres de la Unión Europea y contiene una serie de recomendaciones

Progress report); en 2013 (A Compendium of Case Studies on Gender Mainstreaming Initiatives in UN-Habitat, Housing and Slum Upgrading: Gender issue guide, Gender Responsive Urban Planning and Design: Gender issue guide, Gender and Prosperity of Cities: State of Women in Cities 2012/2013, Gender and Urban Planning); en 2012 (Les Formules de Tenure Partagee pour les Femmes, Evaluation of Gender Mainstreaming in UN-HABITAT, Gender and Economic Development: Global Urban Economic Dialogue Series) y en 2011 (Designing and Evaluating Land Tools with a Gender Perspective, Improving Gender Equality and Grassroots Participation through Good Land Governance: A training package). 
concretas que deben tenerse en cuenta para promocionar de forma creciente la participación de las mujeres en la ciudad.

La Carta inicia una línea de acción que llega hasta la actualidad y constituye un documento de referencia en las medidas para responder a los cambios habidos en la familia, en los usos del tiempo, y en la organización de la ciudad. Por ejemplo, ha destacado que la planificación urbana sigue considerando la familia nuclear con el hombre trabajando fuera y la mujer en el hogar como modelo de referencia, cuando éste ya no es el mayoritario. Señala, además, que las mujeres tienen necesidades propias en relación al espacio urbano que se derivan, fundamentalmente, de su dedicación a las tareas de mantenimiento del hogar, de la atención de niños y ancianos o de la doble jornada laboral y doméstica. Para atender tanto a los cambios en las relaciones de género producidos en las últimas décadas, como al hecho de que las mujeres aún continúan haciéndose cargo de las labores del cuidado en mayor medida que los varones, es necesaria una planificación urbana flexible y coherente con los nuevos modelos sociales y familiares, que facilite la igualdad de oportunidades para las mujeres y los hombres en la vida urbana.

La Carta muestra, así, el mismo compromiso con la política de la diferencia de género que veíamos en el ámbito de Naciones Unidas, al considerar que el objetivo de la igualdad no se logra mediante unas políticas universalistas, sino que pasa por atender a las percepciones y necesidades específicas de las mujeres. La carta incluye doce recomendaciones que deben incorporar los estados miembros con el fin de atender en la planificación de los espacios urbanos a la experiencia que las mujeres tenemos de la ciudad, que es diferente de la de los hombres por tener heterogéneas necesidades de uso (Sánchez de Madariaga, 2004: 17-20), estilo de movilidad (Tobío, 1995: 55-72), o distintas percepciones de seguridad (Román y Velázquez, 2008: 56).

Las recomendaciones de la carta a partir de esta "filosofía de la diferencia" pueden resumirse en cuatro:

En primer lugar, la necesidad de participación de las mujeres en la toma de decisiones, tanto a nivel institucional como en foros intermedios de observación de las dificultades cotidianas, con el fin de lograr una ciudadanía activa. Para ello se requiere "convertir en tema político las condiciones de vida cotidiana analizadas desde la perspectiva de las mujeres (Carta 1996, principio 5). En este sentido, es interesante, por su novedad, la propuesta de potenciar redes de trabajo y niveles intermedios de toma de decisiones que impliquen principalmente a las mujeres y el fomento de asociaciones vecinales alternativas. 
En segundo lugar, es preciso atender a las condiciones de seguridad y movilidad, facilitando "el acceso al transporte para poder desplazarse libremente y con toda seguridad" (Carta 1996, principio 7). Para ello es preciso recoger el punto de vista de las mujeres con el fin de hacer efectivo el "derecho a la ciudad".

El tercer grupo de recomendaciones se refiere a la proximidad de los servicios para hacer más llevaderas las obligaciones que siguen recayendo sobre las mujeres. Un requisito fundamental será, una vez más, atender "a las necesidades manifestadas por las mujeres" (Carta 1996, principio 8).

El último grupo de recomendaciones tiene que ver con lo simbólico. Por un lado, plantea la necesidad de formación de los agentes implicados en la planificación urbana con el fin de que tomen en consideración el factor género, así como lanzar programas de investigación orientados hacia la acción. Además, señala la necesidad de actuar sobre los medios de comunicación y la opinión pública con el fin de combatir los estereotipos de género.

Del somero recorrido realizado hasta aquí podemos concluir que existe todo un marco de recomendaciones a nivel internacional que persiguen la inclusión del género de forma transversal en las políticas públicas de planificación de las ciudades. De cara a sistematizar el análisis de los planes estratégicos, podrían resumirse en los siguientes aspectos:

1. La necesidad de fomentar la participación para atender al punto de vista de las mujeres con el fin de diseñar ciudades que respondan a las necesidades "encarnadas", lejos del individuo abstracto liberal al que nos referíamos al inicio de este artículo.

2. Las desigualdades que afectan de manera especial a las mujeres las sitúan en una posición de desventaja en cuanto al acceso a la vivienda. Por lo tanto, no bastan las políticas universales, sino que es necesario elaborar algún tipo de medida especial que garantice la igualdad en el acceso.

3. La pertinencia de prestar atención a la seguridad o percepción de inseguridad, como un obstáculo para que las mujeres participen en el espacio público en condiciones de igualdad.

4. Conscientes de que muchas de las desigualdades en la participación en la ciudad hunden sus raíces en las condiciones de la vida privada y, sobre todo, en el hecho de que las tareas del cuidado aún recaen en mayor medida sobre las mujeres, es preciso atender a los equipamientos y transportes públicos que faciliten la movilidad haciendo porosas las 
fronteras entre lo público y lo privado establecidas por el orden político liberal.

5. Y, finalmente, la formación en igualdad y la visibilidad simbólica de las mujeres en las ciudades, todo ello, con el fin de hacer "ciudades conciliadoras".

En definitiva, los documentos analizados muestran la necesidad de romper con la separación de espacios sobre la que se han construido las ciudades como reflejo del orden político liberal. Ahora bien, en último término, son las instituciones locales las que deberán materializar esas recomendaciones en sus respectivos planes de desarrollo urbano. De ahí la pertinencia de analizar en qué medida los planes estratégicos de las ciudades han incluido la perspectiva de género y se han hecho eco de las recomendaciones dictadas al respecto por los organismos internacionales que hemos presentado aquí ${ }^{4}$.

\section{LA RECEPCIÓN DE LAS RECOMENDACIONES DE GÉNERO EN LOS PLANES ESTRATÉGICOS DE DESARROLLO URBANO}

Si bien el análisis de los planes estratégicos territoriales se puede enmarcar dentro de la evaluación de las políticas públicas, sus especiales características, como su carácter público-privado, su condición multidisciplinar o el hecho de que sean planes indicativos y no de obligado cumplimiento, hacen que las metodologías y herramientas utilizadas en la evaluación de las políticas públicas no sean, en muchas ocasiones, fácilmente aplicables (Herrador y Martín Mesa, 2010: 120). Conscientes de esta limitación (y a pesar de ella), nos proponemos analizar en qué medida los planes estratégicos de desarrollo urbano han incluido la perspectiva de género. Dada la imposibilidad de estudiar todos y cada uno de los planes elaborados hasta el momento en España, hemos seleccionado los de Bilbao, Barcelona, Zaragoza, Málaga y Jaén. La elección de Barcelona, Bilbao, Málaga y Zaragoza se debe a que son algunas de las ciudades que han tenido en los últimos años una mayor transformación económica y social y han dispuesto de un plan estratégico, continuando dichos planes en la actualidad (Pascual y Tarragona, 2009: 26). Con respecto a Jaén y Málaga, porque en ambos casos los planes estratégicos se han mostrado muy activos y poseen dos cátedras diferentes de planificación estratégica territorial, con la Universidad de Jaén $^{5}$ y con la Universidad de Málaga ${ }^{6}$, respectivamente.

\footnotetext{
${ }^{4}$ Sería de gran interés analizar la recepción de estas recomendaciones a nivel estatal y autonómico. Por razones de espacio no es posible hacerlo aquí.

${ }^{5} \mathrm{http}: / /$ blogs.ujaen.es/catpetdlg/?cat $=1$
} 
En cuanto al ámbito territorial de los planes, analizamos tres de área metropolitana (Bilbao, Barcelona y Zaragoza) porque en esos casos no hay plan de ciudad. En cuanto a Málaga y Jaén, hay plan estratégico tanto de ámbito ciudad como provincial, y en ambos nos hemos centrado en el provincial. En el caso de Jaen, porque el plan de ciudad ha decaído, en el de Málaga porque no aparece ninguna referencia al género, como luego explicaremos con más detalle ${ }^{7}$. Con respecto a la temporalidad del estudio, hemos seleccionado el plan más reciente. Todos ellos están redactados entre el año 2009 y el 2011.

Como metodología utilizada, realizaremos un análisis del contenido de los planes estratégicos y urbanísticos, utilizando como indicadores de la inclusión de la perspectiva de género las recomendaciones principales de los organismos internacionales señaladas en el epígrafe anterior y que hemos tratado de agrupar y sintetizar al máximo. Para ello, llevaremos a cabo dos tareas: en primer lugar, buscar términos como género, igualdad, mujer, conciliación, participación. Posteriormente analizaremos el contenido de la documentación para ver si contiene referencias a alguna actuación concreta -aunque sea únicamente de manera propositiva- en el ámbito de las recomendaciones propuestas.

Hemos señalado antes las dificultades que entraña la evaluación de los planes estratégicos. Realizar un diagnóstico de la inclusión de la perspectiva de género solamente desde el análisis de los planes podría dar una imagen parcial de la preocupación real de las entidades locales con las cuestiones de género. Por eso, en varios casos hacemos referencia a otro tipo de medidas -como el diseño institucional de los ayuntamientos o la elaboración de dictámenes sobre los planesque, aunque no sean el objeto directo de nuestro análisis, muestran la existencia de un compromiso con el género a pesar de que no se recoja expresamente en los planes estratégicos.

El siguiente cuadro presenta de forma preliminar y sistemática los cinco aspectos en los que se pueden resumir las recomendaciones sobre urbanismo analizadas en el apartado anterior y señala si aparece alguna referencia a ellas en los planes estudiados. Pretendemos mostrar aquí una visión general de la inclusión o no

\footnotetext{
${ }^{6} \mathrm{http}: / /$ www.uma.es/catedras/info/4630/catedra-de-planificacion-territorial-ygobernanz $\% 20 \% 20 \mathrm{a}-$ local/

${ }^{7}$ Somos conscientes de que la comparación entre planes estratégicos provinciales y planes urbanos en un tema tan sensible como es el género puede suponer algún tipo de distorsión en el valor de la comparabilidad. Por ello, los resultados y las conclusiones a las que se llegan no deben ser tomadas como irrefutables, sino como propuestas para profundizar en la respuesta a la pregunta por la inclusión de la perspectiva de género en las políticas de planificación urbana.
} 
de dichas recomendaciones, para pasar posteriormente a analizar con más detalle la manera en que las recogen los planes objeto de nuestro análisis.

Cuadro 1. La inclusión de las recomendaciones sobre urbanismo y género en los planes estratégicos de desarrollo urbano

\begin{tabular}{|c|c|c|c|c|c|}
\hline & $\begin{array}{c}P E \\
\text { Barcelona } \\
\text { Metropoli- } \\
\text { tana }\end{array}$ & $\begin{array}{c}\text { PE Bilbao } \\
\text { Metropo- } \\
\text { litano }\end{array}$ & $\begin{array}{c}\text { PE } \\
\text { provincia } \\
\text { de Jaén }\end{array}$ & $\begin{array}{c}\text { PE } \\
\text { provincia } \\
\text { de Málaga }\end{array}$ & $\begin{array}{c}\text { Zaragoza y } \\
\text { su entorno }\end{array}$ \\
\hline $\begin{array}{c}\text { 1. Participación de las } \\
\text { mujeres en el diseño de la } \\
\text { ciudad }\end{array}$ & & & $\mathrm{X}$ & $\mathrm{X}$ & $\mathrm{X}$ \\
\hline $\begin{array}{c}\text { 2. Igualdad en el acceso a la } \\
\text { vivienda }\end{array}$ & $\mathrm{X}$ & $\mathrm{X}$ & $\mathrm{X}$ & $\mathrm{X}$ \\
\hline $\begin{array}{c}\text { 3. Necesidad de seguridad } \\
\text { para el uso del espacio } \\
\text { público }\end{array}$ & & $\mathrm{X}$ & $\mathrm{X}$ & $\mathrm{X}$ & $\mathrm{X}$ \\
\hline $\begin{array}{c}\text { 4. Promover la mejora de } \\
\text { equipamientos y transportes } \\
\text { públicos }\end{array}$ & & & $\mathrm{X}$ & $\mathrm{X}$ & \\
\hline $\begin{array}{c}\text { 5. Incrementar la formación } \\
\text { en igualdady visibilizar a } \\
\text { las mujeres }\end{array}$ & & & & & \\
\hline
\end{tabular}

Fuente: elaboración propia

\subsection{Plan estratégico metropolitano de Barcelona}

El Plan Estratégico Metropolitano de Barcelona es un proyecto promovido por el Ayuntamiento de Barcelona, que integra 36 municipios que conforman el área metropolitana de Barcelona y viene a sustituir a los anteriores planes de ciudad elaborados desde 1990 (Pascual y De Forn, 1999). El primer plan data de 2003, fue 
revisado en 2007 y en 2010 se aprobó el Plan Estratégico Metropolitano de Barcelona Visión 2020, que es el que vamos a analizar aquí.

En el Plan Estratégico Metropolitano de Barcelona Visión 2020 no aparecen ni la palabra "mujer", ni "género", y una sola vez la palabra "igualdad", en relación con la igualdad de oportunidades. Si que contiene varias referencias a una ciudad "humanista, sostenible y responsable", "que ofrezca una mayor calidad de vida" y a la necesidad de atender a "la nueva diversidad ciudadana", una "ciudad abierta y acogedora", "inclusiva y solidaria", que podrían haber servido como punto de anclaje para un mayor desarrollo de una filosofía de la diferencia que incluya la perspectiva de género tal y como la estamos contemplando aquí.

Por otra parte, de las cinco recomendaciones señaladas, no aparece participación, ni percepción de seguridad, ni formación/visibilización de las mujeres. Recoge las que se refieren a acceso a la vivienda y al transporte: se propone "elaborar un plan de promoción del alquiler asequible y digno que facilite la vivienda a los colectivos más vulnerables de la sociedad", que lo entendemos como igualdad en el acceso a la vivienda. Igualmente, propone incrementar los índices de cobertura del transporte público, pero no lo relaciona ni con el género ni con la conciliación.

A pesar de que el Plan no incluya referencias al género ni haya extraído todas las potencialidades que encierran algunos de los valores que lo inspiran y que habrían permitido una apuesta por la política de la diferencia -al menos como declaración de principios- existen un serie de informes sobre el Plan que sí contemplan la diferencia de género desde la perspectiva del marco de nuestro estudio. Cabe destacar el primero de los informes, elaborado por la Comisión de Estrategia del plan, que tiene por título La conciliació de la vida laboral, familiar $i$ personal (Chinchilla y Poelmans, 2004). Dicho informe recomienda un crecimiento ordenado, evitando la creación de nuevas zonas carentes de transportes públicos y comunicaciones y poner en marcha un organismo permanente y transversal, el Observatorio Metropolitano de la Conciliación. Considera que una de las principales condiciones para compatibilizar vida familiar y laboral es liberar tiempo para dedicarlo a la familia. Para ello propone medidas como aplicar el horario europeo o fomentar el trabajo a tiempo parcial (Chinchilla y Poelmans, 2004).

Hay, asimismo, otros informes de la Comisión de Estrategia de interés sobre el tema objeto de estudio, como son el relativo a El uso social del tiempo en la Región Metropolitana de Barcelona, o el que se refiere a La Gestión de la Movilidad en el Área Metropolitana de Barcelona, que sí hacen referencia al género. Todo ello es indicativo de que, a pesar de no recoger de manera expresa nada más que la recomendación referida a los transportes, sí que hay una 
preocupación por la conciliación. Ejemplo de ello -aunque un análisis exhaustivo supondría ir más allá de nuestro objeto de estudio- es el hecho de que desde 2004 el Ayuntamiento de Barcelona desarrolla proyectos relacionados con el uso del tiempo. Cabe destacar la introducción de horarios extensos en equipamientos como las bibliotecas o los polideportivos, servicios como los patios abiertos o el impulso de infraestructuras que favorecen el tiempo personal de diversos sectores de la población, como los caminos escolares o el carril bici. No en vano es la única ciudad del Estado cuyo Ayuntamiento tiene una Concejalía sobre Nuevos Usos Sociales del Tiempo, siendo un área de gran actividad, que desarrolla proyectos específicos para facilitar la conciliación.

\subsection{Plan estratégico de Bilbao metropolitano}

El Plan Estratégico de Revitalización del Bilbao Metropolitano comenzó en 1989. El Plan preveía la existencia de una organización que, agrupando a empresas privadas y Administraciones Públicas, impulsara el Plan como instrumento revitalizador del Bilbao Metropolitano (Martínez Cearra, 1993). Así, en 1991 nacerá la Asociación para la Revitalización del Bilbao Metropolitano (Bilbao Metropoli-30) con la función de poner en marcha las estrategias prioritarias del Plan de Revitalización ${ }^{8}$.Tras una serie de planes y reflexiones elaborados desde entonces y en el contexto de la celebración de su XX aniversario, Bilbao Metropoli-30 publica el documento Bilbao Metropolitano 2030: Es tiempo de profesionales, donde se establecen los principios orientativos y los ejes de acción para los próximos años (Bilbao Metropoli-30, 2011).

En dicha reflexión no encontramos alusiones a los términos buscados ("género", "igualdad", "mujer", "conciliación"). Con respecto a las cinco recomendaciones consideradas en esta investigación, sí aparece "participación", pero referida a la colaboración público/privado, a participación social o a participación en grupos y redes, no a la participación de las mujeres en el diseño de la ciudad. Sin embargo, -como señalábamos antes- el documento no es totalmente indicativo, ya que a pesar de no incluir las recomendaciones internacionales en los planes, desde Bilbao Metropoli-30 se viene trabajando en igualdad de oportunidades desde el año 2000 en la red Parekatuz de la Diputación Foral de Bizkaia (red abierta a todas aquellas entidades que deseen implicarse en el impulso de la igualdad de oportunidades entre mujeres y hombres). Este hecho le ha permitido obtener en 2010 el reconocimiento de Entidad Colaboradora en Igualdad

${ }^{8}$ www.bm30.es 
de Oportunidades entre Mujeres y Hombres por parte del Instituto Vasco de la Mujer-Emakunde, por considerar que la Igualdad de Oportunidades constituye un eje transversal que debe estar presente en el desarrollo económico y en la cohesión social y, por tanto, en el desarrollo de la ciudad. Asimismo, ha creado el grupo de trabajo de "Mujeres Profesionales" dentro del proyecto de profesionalidad BasquePRO, con el objetivo de fomentar que el entorno favorezca el desarrollo profesional de las mujeres. También ha participado como secretaría técnica del Ayuntamiento de Bilbao en el Consejo de las Mujeres de Bilbao por la Igualdad, y ha liderado los programas "Acompañamiento, formación y creatividad para mujeres emprendedoras" e "Intercambio de experiencias y buenas prácticas entre entidades que trabajan en Bizkaia para el apoyo a mujeres emprendedoras", del Departamento de Promoción Económica de la Diputación Foral de Bizkaia. Todas estas tareas demuestran la preocupación de Bilbao Metropoli-30 por los valores para el desarrollo de la ciudad y otros intangibles, como ha señalado Pascual (2007), considerando la igualdad de oportunidades, como valor transversal necesario para el desarrollo de la ciudad.

\subsection{Plan estratégico de Jaén}

El Plan Estratégico de la ciudad de Jaén se inició en septiembre de 2009 con la firma de un convenio entre el Ayuntamiento y la Universidad. Su redacción final data de abril de $2011^{9}$. Uno de los aspectos dignos de destacar del plan es que considera la perspectiva de género como un eje transversal, estando presente en todos los ámbitos: hombres y mujeres de Jaén han de compartir espacios públicos y privados, decisiones y recursos. Un requisito imprescindible para ello es la posibilidad de conciliar la vida personal y laboral. Por otra parte, incluye mención a las cinco recomendaciones analizadas en este estudio, aunque sin relacionarlos con el género. Sin embargo, no lo hemos tomado en consideración, porque no se aprecia actividad en su página web desde la fecha de redacción final y porque en noticias en prensa aparece que el plan finalmente ha decaído ${ }^{10}$. Por este motivo, hemos analizado el Plan Estratégico de la provincia de Jaén, liderado por la Fundación "Estrategias para el desarrollo económico y social de la provincia de Jaén", que inició su andadura en 1997 y continúa vivo en la actualidad ${ }^{11}$. En febrero de 2009 el Patronato de la Fundación aprobó la puesta en marcha del II Plan Estratégico de la provincia de Jaén, la llamada "Estrategia Jaén 2020," dando continuidad al proceso de planificación estratégica iniciado en la provincia en 1997.

\footnotetext{
${ }^{9}$ http://www.jaen2015.es/

${ }^{10} \mathrm{http}$ ://andaluciainformacion.es/jaen/267376/el-ayuntamiento-olvida-el-plan-estratgico-dela-ciudad/

${ }^{11} \mathrm{http}: / /$ www.planestrajaen.org/
} 
Desde la importancia de facilitar la conciliación de la vida laboral, personal y familiar, así como la necesidad de fomentar la participación de la sociedad civil para lograr la igualdad, en la Estrategia Jaén 2020 están ampliamente tratados el primer y el quinto indicador, con referencias a la participación de las mujeres, la necesidad de visibilizarse y formarse, por medio de talleres de sensibilización o con la creación del Consejo Provincial de Igualdad y Participación de las Mujeres Jienenses. El Plan pretende crear un espacio de diálogo, a través de las entidades y las asociaciones provinciales de mujeres, con el doble objetivo de asegurar la participación y visibilización del movimiento asociativo de este colectivo y de coordinar todas las acciones que tengan como fin el fomento de la participación femenina en la vida pública.

El segundo indicador (igualdad en el acceso a la vivienda) se recoge en la recomendación "Pacto por la vivienda". Se trata de poner en marcha una política orientada a atender las necesidades sociales en este ámbito (discapacidad, víctimas de la violencia familiar, precariedad económica, etc.), por lo que se han de establecer con los ayuntamientos pactos locales por la vivienda, donde se contemple la disponibilidad de suelo, medidas para agilizar los trámites en las calificaciones, precios adaptados al territorio y el control de calidad de las viviendas.

La recomendación que se refiere a la dotación de equipamientos y servicios para favorecer la conciliación y la corresponsabilidad está ampliamente presente a lo largo del plan. Concretamente, para lograr la igualdad y la inclusión social, contempla la necesidad de "favorecer la conciliación de la vida personal y laboral", con actuaciones como el pacto provincial por la conciliación (se trata de dotar a las entidades locales de herramientas que permitan el desarrollo y la puesta en marcha de medidas de conciliación a nivel municipal). Asimismo, se plantea la creación de un espacio para el encuentro entre los entes locales, las empresas y los agentes sociales y el intercambio de experiencias o la red de servicios para la corresponsabilidad, que consiste en crear ludotecas o una red de centros mancomunados de educación infantil para menores de tres años. La finalidad de estos espacios, que tendrían horarios compatibles con las jornadas de trabajo de las personas de la unidad de convivencia, es profundizar en la realización del principio de corresponsabilidad en todo aquello relacionado con el cuidado de los miembros de la unidad familiar. Igualmente, persigue el desarrollo de planes de transporte metropolitanos y de planes de movilidad, como instrumento de ordenación y coordinación de los transportes en el interior de cada uno de los ámbitos territoriales (Fundación Estrategias para el desarrollo económico y social de la provincia de Jaén, 2012). Finalmente, el único indicador no encontrado es el tercero, el de la percepción de seguridad. 
En definitiva, aunque sin vinculación con la planificación urbana, las cuestiones relativas al género están reiteradamente tratadas en el plan. Muy posiblemente el enfoque tan amplio sobre la igualdad y la participación de las mujeres sea una de las motivaciones por las que se considera un plan estratégico de segunda generación, que son aquellos que se plantean impulsar la gestión relacional, facilitando la colaboración, la relación, el intercambio y el consenso (Pascual, 2007; Martín Mesa y Merinero, 2010).

\subsection{Plan estratégico de Málaga}

La realización de la Planificación Estratégica de la ciudad de Málaga está llevada a cabo por la Fundación Ciedes, creada en $1994^{12}$. Málaga ciudad se encuentra en su II Plan Estratégico, revisado y reformulado en el documento Nuevos Rumbos con el horizonte de 2020 (Fundación Ciedes, 2010). En el Plan Estratégico de la ciudad de Málaga no aparece ninguna referencia a las cinco recomendaciones objeto de este estudio, ni se encuentran los términos buscados. Por ello, -y, una vez más, con el fin de evitar una respuesta reduccionista a la pregunta por la inclusión de la perspectiva de género- hemos analizado también el plan de la provincia de Málaga.

La Fundación MADECA (Málaga, Desarrollo y Calidad) ${ }^{13}$ es la entidad encargada del diseño y la gestión del plan de desarrollo provincial. El II Plan de Actuaciones Estratégicas de la Provincia de Málaga (2010-2011) es, en realidad, un conjunto de planes de acción en distintos ámbitos, elaborados a partir de una serie de informes y dictámenes sobre la realidad socioeconómica de la provincia realizados en colaboración con la Diputación Provincial. El más relevante para nuestro estudio es el dictamen Género en la provincia de Málaga: Análisis transversal de la igualdad, de septiembre 2010, que incluye varios bloques: tolerancia cero en la violencia de género, feminización de la pobreza, segregación de mujeres y hombres en las opciones educativas y en la tecnología, desigualdad en el mercado de trabajo, reparto de la carga total de trabajo y de los tiempos de dedicación a los ciudadanos. Este último aspecto resulta interesante para nuestro estudio. El Dictamen señala que "la conciliación de la vida personal, familiar y profesional es un asunto de conciliación de tiempos: de tiempo de trabajo y de tiempo disponible para la vida privada". Pero, no solo el uso, sino que también la visión que varones y mujeres tienen del tiempo es diferente: "una lectura plana del

\footnotetext{
12 http://www.ciedes.es/

${ }^{13}$ Aun siendo una Fundación desde marzo de 2011, la asociación se constituyó en abril de 2005 , tras haber trabajado desde 2001 por convenios de colaboración en las actuaciones estratégicas para la provincia. http://www.fundacionmadeca.es/
} 
tiempo de trabajo y del tiempo para la vida privada no permite visibilizar correctamente cuan distintos son, por razones de género, la vivencia cuantitativa y cualitativa por parte de hombres y mujeres, respectivamente, del tiempo de trabajo y del tiempo dedicado a actividades privadas. Para muchos varones el tiempo de trabajo es sobre todo el equivalente al tiempo que dedican a su empleo, mientras que para muchas mujeres el tiempo de trabajo es el tiempo que dedican al empleo y al cuidado del hogar y la familia" (Fundación MADECA, 2010). El plan ha ido, así, al núcleo del problema que señalábamos en el marco teórico de nuestro estudio, al afirmar que para lograr la conciliación es necesario "cuestionar (mínimamente) la raíz del problema, que no es otra que la impronta androcéntrica del sistema productivo, un sistema que finge ser sordo y ciego ante el hecho de que existe un mundo reproductivo o doméstico que le sirve de apoyatura y del cual no puede prescindir" (Fundación MADECA, 2010).

A partir de aquí, propone desarrollar estudios comarcales sobre el uso del tiempo de mujeres y hombres con el fin de elaborar propuestas de compatibilización de los ritmos de las localidades con los de las familias (Fundación MADECA, 2010). Como medidas concretas sugiere "el aumento del número de guarderías, de comedores escolares, de unidades de estancia diurna, de residencias públicas para las personas mayores, servicios de teleasistencia y de ayuda a domicilio en todos los municipios, programas de respiro familiar, programas de salud destinados a cuidar al cuidador y la paulatina provisión de ayudas económicas para la compensación de la labor de las/os cuidadadoras/es informales" (Fundación MADECA 2010).

Siendo muy interesantes y completas todas las recomendaciones del Dictamen, en ningún caso se llega a establecer una relación clara entre la conciliación y la planificación urbana, más allá de las medidas para facilitar la atención de la población dependiente señaladas en el epígrafe anterior. Ni siquiera en los dictámenes de Movilidad y Accesibilidad o de Sostenibilidad y espacios naturales, que sí tratan de promover la mejora de equipamientos y transportes públicos y la sostenibilidad, pero sin relacionarlo con la conciliación. Igualmente, se contempla la segunda recomendación (igualdad de acceso a la vivienda) pero tampoco en este caso se relaciona con el género.

Lo que sí se ha de subrayar del Plan Estratégico de Málaga Provincia es su enfoque transversal, muy probablemente, debido a su punto de vista participativo. Igual que en el caso de Jaén, se trata de un plan estratégico de segunda generación, como así se define en su web ${ }^{14}$, que trata de favorecer la participación e implicación ciudadana (Pascual, 2007; Martín y Merinero, 2010). Asimismo, hay que destacar

\footnotetext{
${ }^{14} \mathrm{http}: / /$ www.fundacionmadeca.es/que-es/19-descripcion-plan-estrategico-general
} 
que tiene abundantes propuestas concretas, con tiempos y actores asignados a cada una de ellas, para conseguir la igualdad de género en la provincia. Además de las señaladas anteriormente, cabe destacar la realización de estudios que cuantifiquen la vulnerabilidad social y la feminización de la pobreza, estudios que cuantifiquen y describan la contribución del trabajo invisible de las mujeres a la economía de la provincia, estudios comarcales y locales sobre el uso del tiempo de mujeres y hombres. Estos estudios desembocan en acciones concretas, como la elaboración de Planes Municipales de Organización del Tiempo de la Ciudad. Igualmente se propone revisar la idoneidad o no de esas acciones, mediante el Pacto por la Igualdad de Género, firmado en 2005 y revisado en el Dictamen de Igualdad en 2010 .

\subsection{Plan estratégico de Zaragoza}

El plan estratégico de Zaragoza y su Entorno lo lleva a cabo la Asociación para el Desarrollo Estratégico de Zaragoza y su Área de Influencia (Ebrópolis), fundada en $1994^{15}$. Una vez terminado el periodo de vigencia del Plan Estratégico de 2006, propuso un nuevo Marco Estratégico en el que se formulan las grandes líneas que deberían marcar el futuro de la ciudad en el año 2020.

La Estrategia Zaragoza 2020. Ciudad, ciudadanía y cohesión social: una ciudad de las personas establece cuatro ámbitos de trabajo: territorial, económico, sostenibilidad y social, educativo y relacional (Ebrópolis, 2011). El documento marco del ámbito "social, educativo y relacional", que tiene por título Estrategia social, educativa, cultural y relacional, contiene algunas sugerencias relacionadas con el objeto de este estudio y que muestran una orientación de las políticas de planificación urbana acorde con el marco teórico del que parte nuestro trabajo. Así, el documento recoge de manera reiterada el objetivo de lograr "una ciudad cohesionada, igualitaria e integradora de la diversidad". Igualmente, señala que "contar con la percepción y necesidades de mujeres, ancianos, infancia y juventud, personas con discapacidades, hará una ciudad más amable y vivible para todos". A partir de aquí se presentan algunas recomendaciones interesantes como la de "configurar una ciudad con una mirada femenina, potenciadora de la igualdad de género y con una cultura de la superación de roles". Este propósito se traduce en indicaciones muy concretas, como tener en cuenta que las mujeres, por norma general, suelen hacer más uso de los transportes públicos, la dotación de servicios comunitarios (centros educativos, culturales y de salud) "que acerquen a las viviendas todos los recursos y servicios necesarios en entornos urbanos que permitan la interrelación y potencien la cohesión social" o también crear "espacios

\footnotetext{
${ }^{15} \mathrm{http}: / /$ www.ebropolis.es/
} 
relacionales informales de relación social como por ejemplo en las salidas del colegio, los parques, los mercados". En este sentido, aunque no hace una referencia expresa a las mujeres, sí que se propone como objetivo fomentar que los municipios del entorno de Zaragoza no se conviertan en ciudades dormitorio, sino que se desarrollen también como espacios de vida, ocio, interacción social en general. Se trata de "basar el crecimiento de Zaragoza en un modelo urbanístico que priorice la ciudad consolidada frente a los grandes y nuevos desarrollos de la periferia".

Otras recomendaciones que aparecen también en el documento de Estrategia Social son: la primera, participación ("Espacios públicos donde ejercer la ciudadanía. Participación"), la segunda, igualdad en el acceso a la vivienda ("Vivienda y cohesión social. La ciudad habitada"), la tercera, seguridad (menciona que en la ciudad cobra importancia la seguridad), la cuarta, promover la mejora de equipamientos y transportes públicos ("Así desde la planificación urbana se pueden promover los hábitos saludables y la seguridad (transporte, zonas actividad física, etc.)"). Con respecto a la quinta recomendación, el plan hace referencia a la visibilización (buscando una ciudad "potenciadora de la igualdad de género", es decir, una ciudad donde se visibilice a las mujeres) pero no hay alusiones a la necesidad de la formación en igualdad.

Además de incluir todas estas recomendaciones, lo más interesante del plan, en nuestra opinión, es que alude expresamente a la necesidad de una "Planificación espacial adecuada: mejora urbanística, tipo de ciudad compacta y multifuncional, accesibilidad y transporte, vivienda", entreviendo una relación entre la planificación urbanística, la sostenibilidad y la conciliación. El hecho de haber tenido en cuenta estas cuestiones se debe, sin duda, a que la planificación de la ciudad se ha "remirado" con ojos de mujer, por utilizar la expresión de Mar Rodríguez Beltrán, quien en la introducción del plan destaca que hemos pasado de observar las políticas urbanas desde una ética de la justicia, masculina, a incorporar una ética del cuidado, femenina (Rodríguez Beltrán, 2011; Seisdedos, 2008). Y no solo eso, sino que además de introducir la perspectiva de las mujeres, cabe subrayar que el plan de Zaragoza persigue claramente superar la dicotomía de espacios y su plasmación en el diseño urbano a la que nos referíamos al principio de este artículo, en la medida en que apuesta por "una ciudad compacta, multifuncional, que posibilita la mixtura social y que establece unas relaciones equilibradas con su entorno. Por lo tanto, tiende puentes para salvar las fronteras y las rupturas espaciales, sociales y culturales".

\section{CONCLUSIONES}


La comparación de la inclusión de los cinco ámbitos en los que hemos resumido y sistematizado las recomendaciones de los organismos analizados arroja algunos resultados interesantes para nuestro estudio.

En primer lugar, por paradójico que pueda resultar, porque parece que es la primera cuestión que surge de manera espontánea de la asociación entre urbanismo y género, la recomendación que menos se recoge en los planes estratégicos territoriales es la que se refiere a la necesidad de seguridad para el uso del espacio público. Solo es recogida en el plan estratégico de Zaragoza y su entorno.

Las recomendaciones con mayor resonancia son las referentes a la igualdad en el acceso a la vivienda, promover la mejora de equipamientos y transporte público. Son recogidas respectivamente cada una de ellas por cuatro planes estratégicos.

En cambio, la recomendación relativa a la formación en igualdad y a visibilizar a las mujeres, aparece solamente en dos planes estratégicos: el de Jaén y el de la provincia de Málaga. El poco eco de esta recomendación resulta preocupante, dada la importancia de la formación en igualdad de los agentes encargados de la elaboración de las políticas públicas, en todos los campos, y en este en particular.

Sí que consideramos positiva -desde la perspectiva de lo que constituye el marco teórico de este trabajo- la inclusión en varios planes de la recomendación de fomentar la participación de las mujeres en el diseño de la ciudad. Señalan la necesidad de atender a las necesidades y experiencias de las mujeres, escuchar su voz y mirar la ciudad desde una perspectiva femenina, lejos del universalismo abstracto liberal, que dificulta el acceso de las mujeres en condiciones de igualdad.

En esta línea, hemos apreciado que aquellos planes considerados de segunda generación, por fomentar la participación y el consenso, son los que tienen una sensibilidad mayor para apreciar la igualdad y la participación de las mujeres. Este es el caso de los planes de la provincia de Jaén, provincia de Málaga y Zaragoza y su entorno. Sin embargo, esto no constituye un demérito del resto de los organismos que gestionan planes, puesto que, aunque no hagan una referencia expresa a las recomendaciones analizadas aquí, llevan a cabo otra serie de actividades en campos relacionados con la igualdad. En este sentido, si bien nuestro estudio arroja resultados desiguales en cuanto a la recepción de las recomendaciones en los planes estratégicos de desarrollo urbano, para poder hacer un diagnóstico certero del alcance del compromiso con una política de planificación urbanística diferenciada no basta con el análisis de los planes estratégicos, sino que es preciso mirar más allá y tener en cuenta otro tipo de acciones o instituciones que sí que muestran la 
inclusión de la perspectiva de género o la preocupación por cuestiones que pueden afectar especialmente a las mujeres. Como hemos visto, el ejemplo de Bilbao o de los informes sobre el plan de Barcelona, o el diseño institucional de su ayuntamiento son paradigmáticos en este aspecto. Un análisis exhaustivo de este tipo de acciones queda fuera de este estudio. Aquí sólo apuntamos una línea interesante de investigación que permitiría profundizar en la respuesta a la pregunta por la inclusión de la perspectiva de género en las políticas de planificación urbana.

Finalmente, el interés de nuestro trabajo no es de carácter meramente teórico o intelectual, sino que pretende ser una herramienta que arroje algo de luz sobre la acción futura de las instituciones encargadas de llevar a cabo los planes estratégicos a nivel local. Las políticas de planificación urbana pueden realizar una importante contribución a superar la dicotomía de espacios sociales típica de la sociedad liberal y su plasmación en la ciudad. Hay un principio del plan de Zaragoza que, en nuestra opinión, expresa muy bien cuál debería ser la orientación de este tipo de políticas y que reproducimos aquí para concluir: "Es importante la reformulación de los modelos de intervención y prácticas urbanísticas que, teniendo en cuenta las condiciones espaciales, contemple la heterogeneidad social, la diversidad cultural y las diferencias de género, edades, condiciones económicas y ambientales". Es responsabilidad de las instituciones locales que esto no sea, sin más, una declaración de intenciones sobre el papel, sino que se traduzca en propuestas concretas. Nuestro trabajo termina en el análisis de la recepción de las recomendaciones sobre la perspectiva de género y las ciudades conciliadoras en los planes estratégicos. Como señala el Plan Estratégico de Barcelona, "de las reflexiones, propuestas y medidas que integran esta publicación, hay que pasar a proyectos responsables, con compromisos concretos". Queda para un estudio posterior -sin duda, de gran interés- ver las acciones llevadas a cabo a partir de dichos planes para construir ciudades conciliadoras, ciudades inclusivas y, al mismo tiempo, diversas.

\section{BIBLIOGRAFÍA}

BAUMAN, Zygmunt (2006): Confianza y temor en la ciudad. Vivir con extranjeros. Barcelona: Arcadia.

BECK, Ulrich y BECK-GERNSHEIM, Elisabeth (2001): El normal caos del amor. Las nuevas formas de la relación amorosa. Barcelona: Paidós.

FRASER, Nancy (1987): "Women, welfare and the politics of need interpretation", Hypatia, 2, pp. 103-121.

HERRADOR, Inmaculada y MARTÍN MESA, Antonio (2010): Evaluación y Planificación Estratégica Territorial. En, Martín Mesa, A. y Merinero, R. (dirs.), Planificación estratégica territorial: estudios metodológicos, pp. 117- 
136. Córdoba: Junta de Andalucía, Consejería de Gobernación y Justicia, Dirección General de Administración Local.

INNERARITY, Carmen (2007): "El islam y la república: un conflicto entre dos identidades". Papers, 84, pp. 139-147.

INNERARITY, Carmen (2009): “Comunidades de violencia. Origen y significado de la violencia urbana en los barrios inmigrantes en las ciudades europeas". Anthropos, 222, pp. 160-189.

INNERARITY, Carmen (2013): "El debate sobre el velo islámico en Gran Bretaña. El multiculturalismo liberal y la identidad nacional". Revista de Estudios Políticos, 162, pp. 149-174.

MARTÍN MESA, Antonio y MERINERO, Rafael (dirs.) (2010): Planificación estratégica territorial: estudios metodológicos. Córdoba: Junta de Andalucía, Consejería de Gobernación y Justicia, Dirección General de Administración Local.

MARTINEZ CEARRA, Alfonso (1993): La revitalización de áreas metropolitanas: el caso del Bilbao metropolitano. Boletín de Estudios Económicos, vol. XLVIII, num. 148, pp. 63-71.

MARTINEZ CEARRA, Alfonso, LANDA, Saioa y SANCHO, Ana (2013): Plan Estratégico del Bilbao Metropolitano. En, Garrido, A. y Gándara, G. (eds.), Nuestras ciudades del futuro, pp. 195-206. Barcelona: Erasmus.

MARTINEZ CEARRA, Alfonso, LANDA, Saioa y SANCHO, Ana (2013): Asociación Bilbao Metropoli-30. En, Garrido, A. y Gándara, G. (eds.), Nuestras ciudades del futuro, pp. 217-226. Barcelona: Erasmus.

MUXÍ, Zaida (2006): “Ciudad Próxima. Urbanismo sin Género”. Revista Ingeniería y Territorio, 75, pp. 68-75.

MUXÍ, Zaida et al (2014): Mujeres trabajando. Guía de reconocimiento urbano con perspectiva de género. Barcelona: Comanegra.

MUXÍ, Zaida et al. (2011): “Qué aporta la perspectiva de género al urbanismo?”. Feminismo/s, 17, pp. 105-129.

PASCUAL, José María (2007): La estrategia territorial como inicio de la gobernanza democrática. Los planes estratégicos de segunda generación. Barcelona: Diputación de Barcelona.

PASCUAL, José María y TARRAGONA, Marta (2009): Estrategia Territorial y Gobierno Relacional. Manual para la planificación estratégica de $2^{a}$ generación. Sevilla: Junta de Andalucía, Dirección General de Administración Local.

PASCUAL, José María y DE FORN, Manuel (1999): La estrategia de las ciudades: los planes estratégicos como instrumento: métodos, técnicas y buenas prácticas. Barcelona: Diputación de Barcelona, Oficina Tècnica de Cooperació.

PATEMAN, Carole (1996): Críticas feministas a la dicotomía público/privado. En, Castells, C. (comp.), Perspectivas feministas en teoría política, pp. 31-53. Barcelona: Paidós.

PHILLIPS, Anne (1995): The politics of presence. Oxford New York: Clarendon Press Oxford University Press. 
ROMÁN, Marta y VELÁZQUEZ, Isabela (2008): Guía de urbanismo con perspectiva de género. Murcia: Comunidad Autónoma de la Región de Murcia, Consejería de Política Social, Mujer e Inmigración.

SÁNCHEZ DE MADARIAGA, Inés (2004): Urbanismo con perspectiva de género. Sevilla: Instituto Andaluz de la Mujer.

SÁNCHEZ DE MADARIAGA, Inés (2009): "Vivienda, movilidad y urbanismo para la igualdad en la diversidad: ciudades, género y dependencia". Ciudad y Territorio. Estudios Territoriales, 161-162, pp. 581-598.

SEISDEDOS, Gildo (2008): "Nosotras, las ciudades: estrategias urbanas para una ética del cuidado o qué hacer cuando mis proyectos estrella se convierten en elefantes blancos". Análisis Local, 81, pp. 7-20.

SENNETT, Richard (2011): "Why complexity improves the quality of city life". LSE Cities.

Disponible en: http://files.lsecities.net/files/2011/11/2011_chw_4025_Sennett.pdf

TOBÍO, Constanza y DENCHE, Concha (eds.) (1995): $\bar{E}$ lespacio según el género, ¿un uso diferencial?. Madrid: Dirección General de la Mujer de la Comunidad de Madrid y Universidad Carlos III.

YOUNG, Iris Marion (1996): Vida política y diferencia de grupo: una crítica del ideal de ciudadanía universal. En, Castells, C. (comp.), Perspectivas feministas en teoría política, pp. 99-126. Barcelona: Paidós.

KYMLICKA, Will (1996): Ciudadanía multicultural. Una teoría liberal sobre los derechos de las minorías. Barcelona: Paidós.

\section{Documentales}

Bilbao Metropoli-30 (2011): Bilbao Metropolitano 2030: Es tiempo de profesionales. Disponible en http://www.bm30.es/AG2030_home_es.html

Bilbao Metropoli-30 (2001): Bilbao 2010: $\bar{L}$ a Estrategia. Disponible en http://www.bm30.es/plan/Bilbao2010-LaEstrategia.pdf

Bilbao Metropoli-30 (1999): Bilbao 2010: Reflexión Estratégica. Disponible en http://www.bm30.es/plan/Bilbao2010-ReflexionEstrategica.pdf

Carta Europea de las Mujeres en la ciudad (1996). Disponible en http://habitat.aq.upm.es/dubai/96/bp018.html

Chinchilla, Nuria y Poelmans, Steven (2004): La conciliació de la vida laboral, familiar $i$ personal. Pla Estratègic Metropolità de Barcelona. Disponible en http://www.pemb.cat/wp-

content/uploads/2011/04/1_Conciliacio_vida_laboral1.pdf

Ebropolis (2011): Marco Estratégico Zaragoza 2020. Disponible en $\mathrm{http}: / /$ www.ebropolis.es/web/arbol/interior.asp?idArbol=14\&idNodo=21

Federación Española de Municipios y Provincias (FEMP) (1996): Mujer y Urbanismo: Una recreación del espacio. Madrid: FEMP.

Fundación CIEDES (2010): Nuevos Rumbos. Reflexiones del II PEM para el 2020.

Disponible en http://www.ciedes.es/index.php/el-plan/nuevos-rumbos 
Fundación MADECA (2010): Género en la provincia de Málaga: Análisis transversal de la igualdad. Dictamen. Disponible en http://www.fundacionmadeca.es/images/pdf/Paneles_Estrategicos/Igualdad/DI CTAMEN_GENERO_V4.pdf

Gobierno de Navarra (2006): Diagnósticos para la realización del I Plan de Igualdad de Oportunidades para mujeres y hombres de la Comunidad Foral de Navarra (2006-2010). Disponible en http://www.navarra.es/NR/rdonlyres/3884E59BF5D0-4221-9300-731F3A801180/113859/DiagnIPlanIgualdadCast.pdf

Gobierno de Navarra (2006): I Plan de Igualdad de Oportunidades para mujeres y hombres de la Comunidad Foral de Navarra (2006-2010). Disponible en http://www.navarra.es/NR/rdonlyres/7F7E948E-827F-4ECD-B7C991F83F0AC609/97882/I20Plan20de20Igualdad2.pdf

Gobierno Vasco-Emakunde (2014) VI Plan para la Igualdad de Mujeres y Hombres (2014-2016).

Disponible

en http://www.emakunde.euskadi.net/contenidos/informacion/u72_iv_plan/es_em akunde/adjuntos/VIPlan_final.pdf

Herrador, Inmaculada y Martín Mesa, Antonio (Dirs.) (2012): II Plan Estratégico de la provincia de Jaén, 2020. Jaén: Fundación "Estrategias para el desarrollo económico y social de la provincia de Jaén".

Instituto de la Mujer (2011): Guía de buenas prácticas para promover la conciliación de la vida personal, familiar y profesional desde Entidades Locales de España $y$ Noruega. Disponible en http://www.femp.es/files/566-1011archivo/Guia\%20BP\%20conciliac\%2021\%20exp\%20cast\%20electronic.pdf

Jaén 2015 (2009): Jaén entorno solidario y sostenible. Disponible en http://www.jaen2015.es/sites/default/files/\%5BPE\%20Ja\%C3\%A9n\%5D\%20D ictamen\%20Comisi\%C3\%B3n\%20Soli_daria\%20Sostenible.pdf

Martínez, Pelayo y Fábregues, Carles (2009): Gestió de la mobilitat a l'àrea metropolitana de Barcelona. Pla Estratègic Metropolità de Barcelona. Disponible en http://www.pemb.cat/wp-content/uploads/2011/04/14.Informe_de_Mobilitat.pdf

Ministerio de Fomento (1996): Agenda Habitat España: contribución de las ciudades al desarrollo sostenible. Disponible en http://www.femp.es/files/566-224archivo/Agenda\%20Habitat.pdf

Miralles, Carme (2008): L'ús social del temps a la regió metropolitana de Barcelona. $\mathrm{Pla}$ Estratègic Metropolità de Barcelona. Disponible en http://www.pemb.cat/wp-content/uploads/2011/04/Usos-del-Temps.pdf

Naciones Unidas (1996): Declaración de Estambul sobre los Asentamientos Humanos. Disponible en http://Habitat.aq.upm.es/aghab/adeclestambul.html

Naciones Unidas (1996): Programa Habitat. Disponible en http://habitat.aq.upm.es/aghab/aproghab.html

ONU-HABITAT (2014): Gender Issue Guide: Gender Responsive Urban Research and Capacity Development. en http://unhabitat.org/?wpdmact=process\&did=MTEyMy5ob3RsaW5r 
ONU-HABITAT (2014): Women in Post-Conflict Settlement Planning. Disponible en http://unhabitat.org/?wpdmact=process\&did=MTMzLmhvdGxpbms=

ONU-HABITAT (2013): Planificación y diseño de una movilidad urbana sostenible: Informe Mundial sobre Asentamientos Humanos 2013. Resumen Ejecutivo. Disponible en http://www.onuhabitat.org/index.php?option=com_docman\&task=doc_view\&g $\mathrm{id}=954 \& \mathrm{tmpl}=$ component $\&$ format $=$ raw $\&$ Itemid $=538$

ONU-HABITAT (2013): A Compendium of Case Studies on Gender Mainstreaming Initiatives in UN-Habitat. Disponible en http://mirror.unhabitat.org/pmss/getElectronicVersion.aspx?nr=3488\&alt=1

ONU-HABITAT (2013): Gender and Prosperity of Cities: State of Women in Cities 2012/2013. Disponible en http://mirror.unhabitat.org/pmss/getElectronicVersion.aspx?nr=3457\&alt=1

ONU-HABITAT (2013): Gender Equality Action Plan: Progress report. Disponible en $\mathrm{http}: / /$ mirror.unhabitat.org/pmss/getElectronicVersion.aspx?nr=3507\&alt=1

ONU-HABITAT (2013): Housing and Slum Upgrading: Gender issue guide, Gender Responsive Urban Planning and Design: Gender issue guide. Disponible en http://unhabitat.org/?wpdmact=process\&did=MTgxLmhvdGxpbms=

ONU-HABITAT (2012): Gender and Urban Planning. Disponible en $\mathrm{http} / /$ mirror.unhabitat.org/pmss/getElectronicVersion.aspx?nr=3351\&alt=1

ONU-HABITAT (2012): Les Formules de Tenure Partagee pour les Femmes. Disponible

en http://unhabitat.org/?wpdmact=process\&did=MzIzLmhvdGxpbms $=$

ONU-HABITAT (2011): Designing and Evaluating Land Tools with a Gender Perspective. Disponible en http://unhabitat.org/?wpdmact=process\&did=Mzk2LmhvdGxpbms=

ONU-HABITAT (2011): Evaluation of Gender Mainstreaming in UN-HABITAT. Disponible http://mirror.unhabitat.org/pmss/getElectronicVersion.aspx?nr=3507\&alt=1

ONU-HABITAT (2011): Gender and Economic Development: Global Urban Economic Dialogue Series. Disponible en http://unhabitat.org/?wpdmact=process\&did=MzY0LmhvdGxpbms=

ONU-HABITAT (2011): Improving Gender Equality and Grassroots Participation through Good Land Governance: A training package. Disponible en http://unhabitat.org/?wpdmact=process\&did=Mzk3LmhvdGxpbms=

ONU-HABITAT (2010): Igualdad de género para Ciudades más inteligentes. Desafíos $y \quad$ avances. Disponible en http://unhabitat.org/?wpdmact=process\&did=NDkzLmhvdGxpbms=

ONU-HABITAT (2001): Indicadores Urbanos Estambul +5 . Disponible en $\mathrm{http} / / /$ www.onuhabitat.org/index.php?option=com_docman\&task=doc_downlo ad\&gid $=142 \&$ Itemid $=71$

Pla estratègic metropolitá de Barcelona (2010): El Plan Estratégico Metropolitano de Barcelona - Visión 2020. Disponible en http://www.pemb.cat/es/vision-2020/ 
Rodríguez Beltrán, Mar (2011): Ciudad, ciudadanía y cohesión Social. Una ciudad de las personas. Documento marco del ámbito Social, Educativo y Relacional de la Estrategia Zaragoza 2020. EBRÓPOLIS. Disponible en http://www.ebropolis.es/files/File/Plan\%20Estratgico/ebropolis-estrategia2020social.pdf 